\title{
Overcoming Nanoscale Friction Barriers in Transition Metal Dichalcogenides
}

\author{
Antonio Cammarata* and Tomas Polcar ${ }^{\dagger}$ \\ Faculty of Electrical Engineering, Department of Control Engineering, \\ Czech Technical University in Prague, Technicka 2, 16627 Prague 6, Czech Republic
}

\begin{abstract}
We study the atomic contributions to the nanoscale friction in layered $\mathrm{MX}_{2}(\mathrm{M}=\mathrm{Mo}, \mathrm{W} ; \mathrm{X}=\mathrm{S}$, $\mathrm{Se}, \mathrm{Te}$ ) transition metal dichalcogenides by combining ab initio techniques with group theoretical analysis. Starting from stable atomic configurations, we propose a computational method, named Normal-Modes Transition Approximation (NMTA), to individuate possible sliding paths from only the analysis of the phonon modes of the stable geometry. The method provides a way to decompose the atomic displacements realizing the layer sliding in terms of phonon modes of the stable structure, so as to guide the selection and tuning of specific atomic motions promoting $\mathrm{MX}_{2}$ sheets gliding, and to adjust the corresponding energy barrier. The present results show that main contributions to the nanoscale friction are due to few low frequency phonon modes, corresponding to rigid shifts of $\mathrm{MX}_{2}$ layers. We also provide further evidences that a previously reported Ti-doped $\mathrm{MoS}_{2}$ phase is a promising candidate as new material with enhanced tribologic properties. The NMTA approach can be exploited to tune the energetic and the structural features of specific phonon modes, and, thanks to its general formulation, can also be applied to any solid state system, irrespective of the chemical composition and structural topology.
\end{abstract}

PACS numbers: 63.20.-e, 63.20.D-, 63.20.kg, 63.22.-m, 81.05.Zx

Keywords: friction; sliding; phonon; transition metal dichalcogenides; energy barrier; cophonicity

\section{INTRODUCTION}

Two-dimensional layer materials have been studied for decades thanks to their peculiar chemical and physical properties. Since graphene has recently been successfully prepared and characterized, ${ }^{1}$ its properties have been explored so extensively that research on pristine graphene has already reached its climax, and further developments on graphene-derived compounds are more complex and thus expected at a slower pace. Transiton metal dichalcogenides (TMDs) are a promising alternative to graphene, thanks to their versatile mechanical and electronic properties. The simple stoichiometry of TMDs $\left(\mathrm{MX}_{2}\right.$, where $\mathrm{M}$ is a transition metal with formal charge +4 and $\mathrm{X}$ is a chalcogen of group VIA - S, Se, Te) and their lamellar geometry can be promptly adjusted to build a vast variety of van der Waals heterostructures, ${ }^{2}$ with diverse electronic, ${ }^{3}$ chemical ${ }^{4}$ and mechanical properties. ${ }^{5}$ In the present work, we will focus on the latter, in particular on the atomic contributions to nanoscale friction.

One of the main difficulties in understanding and predicting frictional response is the intrinsic complexity of highly non-equilibrium processes in any tribological contact, which includes the breaking and formation of multiple interatomic bonds between surfaces in relative motion. Understanding the physical nature of these instabilities is crucial to properly describe friction mechanisms. Current ab initio studies already present in the literature describe the atomic origin of tribological properties in terms of energy landscape variations as a function of geometric parameters, e.g. reciprocal position and orientation of two subsequent $\mathrm{MX}_{2}$ layers. ${ }^{6,7}$ In our previous works on the nanoscale friction in $\mathrm{MX}_{2} \mathrm{TMDs}^{8-10}$ we already observed that few low frequency phonon modes are directly related to the sliding of adjacent layers. Such modes correspond to pure rigid layer translations or layer shifts combined with intralayer motions, like stretching and/or bending of atomic bonds, or flattening of coordination polyhedra; finally, based on considerations on the properties of the stable geometry, we argued that the intrinsic friction can be adjusted by a fine control of such modes.

Here we extend our previous work in a substantial way by studying possible potential energy barriers related to the layer sliding in $\mathrm{MX}_{2}$ TMDs, choosing $\mathrm{M}=$ Mo, $\mathrm{W}$ and $\mathrm{X}=\mathrm{S}$, Se, Te. To this aim, we formulate a computational protocol, that we call Normal-Modes Transition Approximation (NMTA), which allows to: $i$ ) find a reasonable guess of possible sliding paths, and $i i$ ) approximate the atomic displacements realizing each path with a combination of phonon modes, in order to promote the sliding and/or tune the related energy barrier. With the expression sliding path we refer to the sequence of geometric transformations which describes the relative parallel shift of two subsequent $\mathrm{MX}_{2}$ layers. An energy barrier is associated to a specific sliding path; such barrier corresponds to the adiabatic contribution to the intrinsic friction hindering the layer gliding. The proposed method requires only the knowledge of the frequencies and displacement patterns forming the complete set of the phonon modes of the stable geometry. The estimation of the sliding path obtained with such approach is then optimized by means of standard techniques. ${ }^{11,12}$ The optimized geometric path and the relative energy profile are then decomposed in terms of phonon modes of the reference stable structure. In this way, we obtain information on which are the atomic motions responsible of the layer sliding (transition path) and which are the electronic and structural features that we have to finely manipulate in order to facilitate the layer sliding, that is to lower the sliding energy barrier. In detail, we identify the main 
contributions to the intrinsic friction with specific few low frequency phonon modes; moreover, we provide further evidences that a particular Ti-doped $\mathrm{MoS}_{2}$ phase previously identified, ${ }^{8}$ is a promising candidate as a novel material with improved frictional properties. Thanks to its general formulation, we expect that the NMTA method can be extended to the study of transition paths and barrier in a vast variety of solid state systems, irrespective of their chemical composition or atomic geometry.

The manuscript is structured in the following way. We first discuss the technical aspects of the NMTA approach; then we show how the method works and which information it can provide. We finally use the NMTA to individuate and study a possible sliding path in the selected $\mathrm{MX}_{2}$ TMDs, discussing which are the features relevant to the layer sliding, and how to harness them to reduce friction in nanoscale TMD-based materials.

\section{THE "NORMAL-MODES TRANSITION APPROXIMATION" METHOD}

In the harmonic approximation, we can write the potential energy $E$ of the system as a sum of $E_{k}$ contributions each coming from a single phonon mode $k$ as ${ }^{13}$

$$
E=\sum_{k} E_{k}\left(\zeta_{k}\right)=\sum_{k} \frac{1}{2} \omega_{k}^{2} \zeta_{k}^{2}
$$

where $E_{k}$ is a function of the normal coordinate $\zeta_{k}$. Following the standard definition of normal modes, ${ }^{13} \zeta_{k}$ is a sinusoidal function of the time $t$ with amplitude $C_{k}$ and associated frequency $\omega_{k}$ :

$$
\zeta_{k}(t)=C_{k} \sin \left(\omega_{k} t+\delta_{k}\right)
$$

where $\delta_{k}$ is the phase determined by the initial conditions. Given a specific phonon mode $k$, the relation between the $i$-th Cartesian displacement $\eta_{i}$ and the normal coordinate $\zeta_{k}$ is

$$
\eta_{i}=a_{i k} \zeta_{k},
$$

where $\left\{a_{i k}\right\}$ is the matrix defining the transformation from Cartesian to normal coordinates; the $k$-th phonon mode thus represents a syncronous displacement of all the atoms at the corresponding frequency $\omega_{k}$ (see "The $E_{k}$ Sinusoidal Form Hypothesis" section of the Supplemental Material).

Let's focus our attention on an arbitrary mode $k$ and consider arbitrary values of the normal coordinate $\zeta_{k}$. From now on, we will consider only those $\zeta_{k}$ value ranges for which the energy $E_{k}$ of the $k$-mode remains bounded, varying as a continuous function between an upper $\left(E_{k}^{\max }\right)$ and a lower $\left(E_{k}^{\text {min }}\right)$ limit $\left(-\infty<E_{k}^{\text {min }} \leq E_{k} \leq E_{k}^{\text {max }}<\right.$ $+\infty$ ); mathematical derivation of what follows is discussed in more detail in "The $E_{k}$ Sinusoidal Form Hypothesis" section of the Supplemental Material. Let's regard the normal coordinate $\zeta_{k}$ as a configurational coordinate. At the stable state, $\zeta_{k}=\zeta_{0}$ and $E_{k}\left(\zeta_{0}\right)=E_{k}^{m i n}$; for simplicity, we can set $\zeta_{0}=0$ and $E_{k}^{\text {min }}=0$. Along the $\zeta_{k}$ coordinate, the energy $E_{k}$ is bounded by hypothesis thanks to the assumption we made above; then there exists $\zeta_{k}^{t r}>\zeta_{0}$ such that $E_{k}\left(\zeta_{k}^{t r}\right)=E_{k}^{\max } \equiv E_{k}^{t r}$ is the only energy maximum in $\left[\zeta_{0}, \zeta_{k}^{t r}\right]$. In such range, we can approximate the potential energy $E_{k}$ of a single $k$-mode with a Fourier Series truncated at the first term as

$$
E_{k}\left(\zeta_{k}\right)=\frac{1}{4} \omega_{k}^{2} C_{k}^{2}\left[1-\cos \left(\frac{\pi}{C_{k}} \zeta_{k}\right)\right],
$$

being the hypotheses required by the Fourier theorem satisfied thanks to our assumptions on the $E_{k}\left(\zeta_{k}\right)$ function.

The geometric configuration $\zeta_{k}=\zeta_{k}^{t r}$, at which the maximum of the energy is realized, can then be regarded as the closest transition state to the stable state $\zeta_{0}$ along the $\zeta_{k}$ configurational coordinate. The $\left[\zeta_{0}, \zeta_{k}^{t r}\right]$ range, which corresponds to that part of the path along the $k$-mode connecting the stable state $\zeta_{0}$ to the closest transition state $\zeta_{k}^{t r}$, is the transition path along which the transition from $\zeta_{0}$ to $\zeta_{k}^{t r}$ is realized. Moreover, since we assumed that $E_{k}\left(\zeta_{k}^{t r}\right)$ is the only maximum in $\left[\zeta_{0}, \zeta_{k}^{t r}\right]$, no metastable states are present between the stable and the closest transition state.

By comparing Equation 1 with Equation 2, and in agreement with Equation 4, we see that the energy $E_{k}^{t r}$ of the transition state

$$
E_{k}^{t r}=\frac{1}{2} \omega_{k}^{2}\left(\zeta_{k}^{t r}\right)^{2}=\frac{1}{2} \omega_{k}^{2}\left(C_{k}^{t r}\right)^{2}
$$

is proportional to the square of the product between an electronic and a geometric factor: $i) \omega_{k}$, the frequency associated to the $k$-mode, which depends on the electronic features of the atomic types; ii) $\zeta_{k}^{t r} \equiv C_{k}$, the normal coordinate value at which the transition is realized, which depends on the geometric features of the system and coincides with the maximum amplitude of the normal coordinate $\zeta_{k}$. The smaller the frequency of the mode, the smaller the energy needed by the system to undergo a transformation from the stable configuration to the transition state. Leaving unaltered the atomic topology, the frequency of the mode can be tuned by changing specific atomic types present in the structure. The selection of suitable atomic types can be done by means of electrostructural descriptors like the cophonicity metric. ${ }^{8-10}$ By substituting specific atomic sites with suitable atomic species, it is therefore possible to tune the energy barrier. Moreover, the transition state can be identified by inspecting how the stable geometry varies by varying the amplitude $\zeta_{k}$; in the practice, the transition state can be numerically evaluated by imposing that the interatomic distances fall in a specific range at varying $\zeta_{k}$. At the transition $\zeta_{k}^{t r}$, the ratios among the atomic displacements define a relation among the atomic positions that can be exploited to estimate the transition state in any system with the same atomic topology but with different chemistry; it is the case, for example, of the $\Gamma$ (4) rigid sliding mode in hexagonal $P 6_{3} / m m c \mathrm{MX}_{2}$ Transition Metal 
Dichalcogenides (TMDs), that we analyse later on in the present study.

Along a general configurational coordinate $\zeta$, the total potential energy $E$ can be approximated as a sum of contributions from all the $K$ normal modes, by expressing $E_{k}$ in a more general form than that shown in Equation 4:

$$
E(\zeta)=\sum_{k=1}^{K} \frac{1}{4} \omega_{k}^{2} C_{k}^{2}\left[1-\cos \left(\frac{\pi}{C_{k}} \zeta-\theta_{k}\right)\right]
$$

where some (or all) of the $C_{k}$ amplitudes and $\theta_{k}$ phases can be correlated if the modes are coupled along $\zeta$.

Approximation done in Equation 4 is reliable only if, along the $\left[\zeta_{0}, \zeta_{k}^{t r}\right]$ transition path, the frequency of all the other modes vary within a small range. The wider such range, the more important the contribution of the other modes to the potential energy along the selected mode; ${ }^{14}$ in that case, the modes are coupled and the expression for $E_{k}$ must take into account any deviation from the harmonicity:

$$
\begin{aligned}
E_{k}\left(\zeta_{k}\right)= & \frac{1}{4} \omega_{k}^{2} C_{k}^{2}\left[1-\cos \left(\frac{\pi}{C_{k}} \zeta_{k}\right)\right]+ \\
& +f\left(\zeta_{k}, \omega_{j}, C_{j}\left(C_{k}\right) ; \forall j \neq k\right)
\end{aligned}
$$

where $f\left(\zeta_{k}, \omega_{j}, C_{j}\left(C_{k}\right) ; \forall j \neq k\right)$ is a function of the amplitudes and the frequencies of all the modes other than $k$, and each amplitude $C_{j}$ of the $j$-th mode is a function of the $C_{k}$ amplitude of the mode $k$. Using Equation 6, the correction $f$ can be approximated as

$$
\begin{array}{r}
f\left(\zeta_{k}, \omega_{j}, C_{j}\left(C_{k}\right) ; j \neq k\right) \approx \\
\approx \sum_{j \neq k} \frac{1}{4} \omega_{j}^{2} C_{j}^{2}\left[1-\cos \left(\frac{\pi}{C_{j}} \zeta_{k}-\theta_{j}\right)\right],
\end{array}
$$

which represents the anharmonic contribution to the $k$ mode.

Each $\zeta_{k}$ normal coordinate can thus be followed to look for possible transition states. In this respect, the complete set of normal modes constitutes a finite number of possible routes to explore the energy landscape in a systematic way. In the practice, a specific $k$-mode is first selected, and the transition path is initially estimated using intermediate geometries connecting the stable and the transition state along the $\zeta_{k}$ coordinate. Subsequently, such geometries can be eventually modified by inducing atomic displacements according to the distortion patterns of modes other than the selected one, in order to capture different subtle and interesting features of the transition. The corresponding total potential energy is thus built as a linear superposition of all the modes considered in the transition: such superposition corresponds to the approximation done in Equation 6, in which only the modes participating to the transition will have non-null $C_{k}$ amplitudes. To explore all the possible transition paths, we then consider the entire set of the $k$-modes, select one at a time, and build the transition path by considering other modes that, eventually, participate to the transition. It is worthy to note here that, the individuation of possible transition paths can be performed by a numerical evaluation of geometries that requires the knowledge of only the displacement patterns of the phonon modes: once the phonon modes of the stable state have been calculated, the estimation of possible transition paths can be computed within few minutes of wallclock time on an ordinary desktop computer. Moreover, despite the number of linear superpositions to consider could be high, such number is still a finite quantity completely defined by the complete set of normal modes. This aspects of the NMTA method drastically reduces the effort to look for possible transition states of a given system, providing a systematic prescription to build possible transition paths.

Once the estimation of the transition path is built and then optimized by means of numerical techniques, ${ }^{11,12}$ Equation 6 can be used to deconvolute the calculated energy profile into contributions of single modes. By comparing the $C_{k}$ amplitudes it is possible to identify which is the mode that leads the geometric transition: the higher the $C_{k}$ value is, the higher the atomic displacement induced by the $k$-mode. Equation 6 thus constitutes a tool to identify how each mode contributes to the advancement of transitions, providing a route to finely tune reaction barriers through the adjustment of the characteristics (frequency and displacements) of single phonon modes.

\section{RESULTS AND DISCUSSION}

In the following sections, we will show how the NMTA method is used to estimate a possible sliding path in the selected $\mathrm{MX}_{2}$ systems, to tune the properties of single phonon modes and how these are related to the sliding potential energy barrier. The technical details of the calculations are reported in the "Technical Details of AbInitio Simulations" section of the Supplemental Material. We also analyse two case studies, the inversion of the ammonia molecule and the $\mathrm{HCN} \rightarrow \mathrm{CNH}$ isomerization reaction of the hydrogen cyanide molecule, in order to show how to individuate a possible transition path from the coupling of multiple phonon modes and how to evaluate their contribution to the transition rate constant. However, we report such discussion in the Supplemental Material, while here we will focus on layer shifts in transition metal dichalcogenides.

\section{A. The Rigid Shift of Subsequent Layers in $\mathbf{M X}_{2}$ Transition Metal Dichalcogenides}

We consider the bulk structure of $\mathrm{MX}_{2}$ transition metal dichalcogenides, with $\mathrm{M}=\mathrm{Mo}, \mathrm{W}$ and $\mathrm{X}=\mathrm{S}$, Se, Te, together with the $\mathrm{Mo}_{3} \mathrm{TiS}_{8}$ system which we reported in our previous studies $;, 9$ for simplicity, we will refer to them as MX and Ti:MoS, respectively. We will here use the NMTA method to individuate a possible transition path 


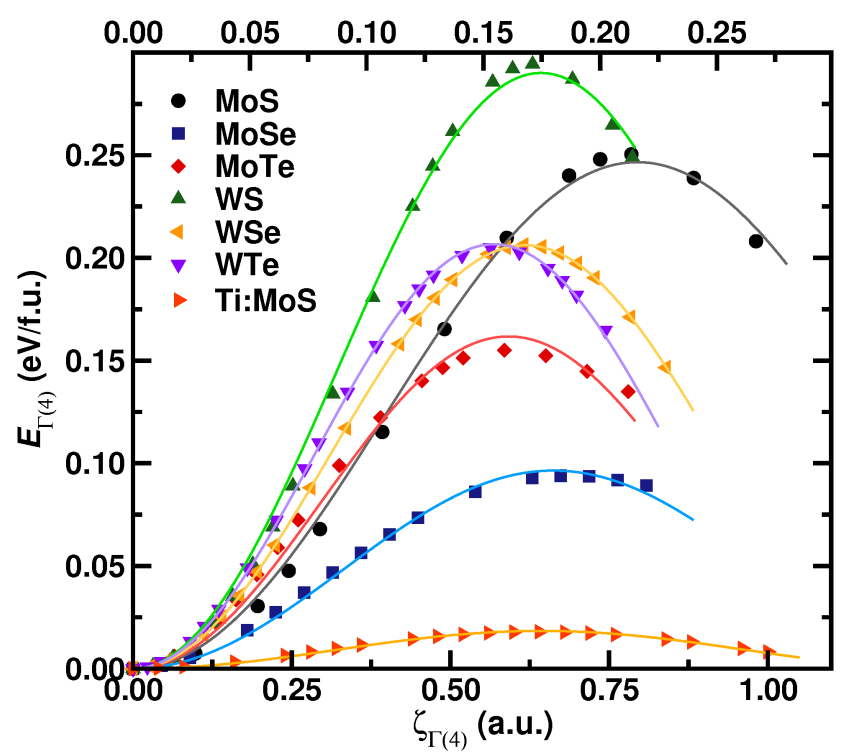

FIG. 1. Calculated energy values (symbols) of the considered compounds along the $\zeta_{\Gamma(4)}$ configurational coordinate, corresponding to the $\Gamma(4)$ vibrational mode. The top and the bottom $x$-axis are the reference for the MX and Ti:MoS systems, respectively. Each solid line represents the best fit of the corresponding data set by means of Equation 4 .

starting from one vibrational mode of the ground phase. As an example, we select the $\Gamma(4)$ phonon mode, corresponding to rigid sliding of subsequent layers (see " $\Gamma(4)$ Rigid-layer Shift Vibrational Mode of MX and Ti:MoS Systems" section of Supplemental Material). We build the transition path along the $\zeta_{\Gamma(4)}$ configurational coordinate in this way: the first structure of the path is the ground state configuration $\zeta_{0}$; we then build the path sequence by distorting $\zeta_{0}$ using gradually increasing values of $\zeta_{\Gamma(4)}$. This can be done either by inspecting the displacement vectors of the $k$-mode and modifying the equilibrium coordinates accordingly by hand, or by using tools like ISODISTORT ${ }^{15}$ or the BILBAO crystallographic server. ${ }^{16,17}$ We then calculate the energy of the considered geometries, in order to estimate the corresponding energy profile. ${ }^{18}$ Inspecting Figure 1, it is immediately apparent that the energy expression in Equation 4 is a very good approximation of the calculated values. To check if the $\Gamma(4)$ mode is coupled with other normal modes, we calculate the phonon band structure along a standard ${ }^{19}$ linear path joining the high-symmetry points of the irreducible Brillouin zone (IBZ) at each of the considered $\zeta_{\Gamma(4)}$ amplitudes. We want to stress here that a standard phonon-phonon coupling calculation would not provide enough information for our purposes, since it is performed considering small displacements about the stable configuration, while we are considering geometries far from the equilibrium. We find that, in the MoSe, MoTe and Ti:MoS systems, the frequency values of the modes calculated at the high symmetry points of the IBZ display similar trend at increasing $\zeta_{\Gamma(4)}$ amplitudes, hence affecting the $E_{\Gamma(4)}$
TABLE I. Calculated mode amplidude $\zeta_{\Gamma(4)}^{t r}$ (a.u.) and corresponding energy maximum $E_{\Gamma(4)}^{t r}(\mathrm{e} . \mathrm{V} /$ f.u. $)$, frequency $\omega_{\Gamma(4)}$ $\left(\mathrm{cm}^{-1}\right)$ and cophonicity $C_{p h}(\mathrm{M}-\mathrm{X})\left(\mathrm{cm}^{-1}\right)$ of each of the considered TMD chemistries. $\zeta_{\Gamma(4)}^{t r}$ and $E_{\Gamma(4)}^{t r}$ values are extracted from the fitting functions shown in Figure 1, while $\omega_{\Gamma(4)}$ and $C_{p h}(\mathrm{M}-\mathrm{X})$ values have been calculated using the stable configuration of the corresponding compound.

\begin{tabular}{lcccc}
\hline \hline System & $\zeta_{\Gamma(4)}^{t r}$ & $E_{\Gamma(4)}^{t r}$ & $\omega_{\Gamma(4)}$ & $C_{p h}(\mathrm{M}-\mathrm{X})$ \\
MoS & 0.22 & 0.25 & 30 & 0.009 \\
MoSe & 0.18 & 0.10 & 28 & -0.034 \\
MoTe & 0.16 & 0.16 & 30 & -0.066 \\
WS & 0.19 & 0.29 & 26 & 0.046 \\
WSe & 0.17 & 0.21 & 24 & 0.024 \\
WTe & 0.15 & 0.21 & 22 & -0.029 \\
Ti:MoS & 0.63 & 0.02 & 22 & -0.005 \\
\hline \hline
\end{tabular}

values in the same way. For this reason, we can compare the corresponding maximum energy values $E_{\Gamma(4)}^{t r}$ among those realized at similar $\zeta_{\Gamma(4)}^{t r}$ amplitudes (Table I); we can appreciate that the energy of the transition state $\zeta_{\Gamma(4)}^{t r}$ is smaller when the corresponding frequency is lower, in a good agreement with Equation 5. Same argument applies to the WS, WSe and WTe systems. We finally note that the MoS system is the only one for which some frequencies, different from $\omega_{\Gamma(4)}$, vary within a range wider than what we observe in the remaining systems. This means that in the MoS system, the contribution to $E_{\Gamma(4)}$ from such modes, corresponding to the correction term $f$ in Equation 7, cannot be neglected and comparison with the other systems is not trivial. This result indicates that $\Gamma(4)$ is strongly coupled with all those modes whose frequency varies along the $\left[\zeta_{0}, \zeta_{\Gamma(4)}^{t r}\right]$ path. In this respect, we can state that the harmonic approximation is valid not only for small displacements about the equilibrium position, but also for any displacement magnitude and pattern for which the modes can be considered uncoupled.

We now want to relate the maximum energy values $E_{\Gamma(4)}^{t r}$ to the dynamical features of the system in the stable geometry. To do this, using the stable configuration, we calculate the cophonicity ${ }^{8} C_{p h}(\mathrm{M}-\mathrm{X})$ of the $\mathrm{M}-\mathrm{X}$ pair in the frequency range $[0,35] \mathrm{cm}^{-1}$, corresponding to the frequency range of the $\Gamma(4)$ mode common to all the considered chemical compositions; we then relate the energy maximum to the calculated M-X pair cophonicity. We exclude the MoS system from the present analysis, due to non-negligible coupling effects we refer above. We observe that the lowest $E_{\Gamma(4)}^{t r}$ value is realized when $C_{p h}(\mathrm{M}-\mathrm{X}) \approx 0.0 \mathrm{~cm}^{-1}$ (Table I and Figure $2 \mathrm{a}$ ), that also realizes the maximum $\zeta_{\Gamma(4)}$ amplitude among the considered compounds (Figure $2 \mathrm{~b}$ ). The cophonicity of the M-X pair can thus be exploited as a knob to adjust the frequency of a mode, the energy of the related transition state and the amount of distortion amplitude needed to build such transition configuration starting from the stable geometry. 


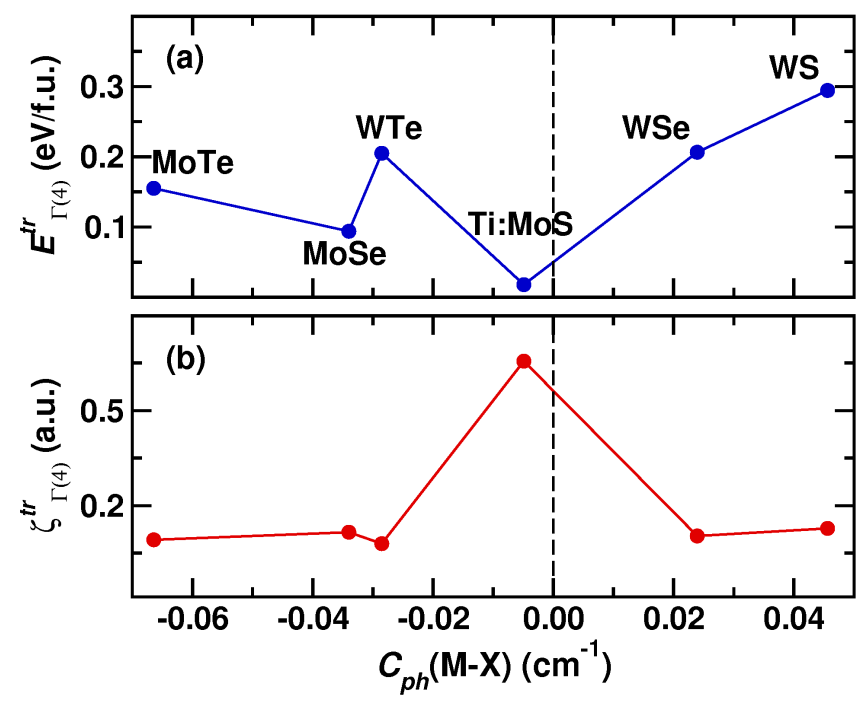

FIG. 2. Energy of the transition state (a) and corresponding $\zeta_{\Gamma(4)}^{t r}$ mode amplitude (b) of each considered compound as a function of the M-X pair cophonicity. Cophonicity values close to zero realize the minimum energy barrier and the maximum amplitude displacement. Lines are guides for the eye.

We now extend our exploration of the transition path along the selected $\Gamma(4)$ mode beyond the corresponding transition state $\zeta_{\Gamma(4)}^{t r}$. According to Equation 4, the closest minimum beyond the transition state is located at $\zeta_{\Gamma(4)}=\zeta_{\Gamma(4)}^{*}>\zeta_{\Gamma(4)}^{t r}$; thus the corresponding energy $E_{\Gamma(4)}\left(\zeta_{\Gamma(4)}^{*}\right)$ is null, as it is for the stable position $\zeta_{0}$. Indeed, irrespective of the eventual coupling with other modes, the assumption that the closest minimum is located at $\zeta_{\Gamma(4)}=\zeta_{\Gamma(4)}^{*}$ does not necessarily implies that the actual energy value $E_{\Gamma(4)}\left(\zeta_{\Gamma(4)}^{*}\right)$ is the same of that pertaining the stable configuration, from which the mode $\Gamma(4)$ has been calculated. This occurs because, in general, the displacement pattern of a $k$-mode is not a periodic function of the mode amplitude. However, if Equation 4 is a good approximation of the energy profile in $\left[\zeta_{0}, \zeta_{k}^{t r}\right]$, as it is the case of $k=\Gamma(4)$ mode in the examined TMD systems, the magnitude of the energy curvature takes very close values at both $\zeta_{0}$ and $\zeta_{k}^{t r}$; that is, the energy minimum, used to calculate the phonon spectrum, and its closest maximum show similar energy curvature values along the selected $k$-mode. Considering the geometry at $\zeta_{\Gamma(4)}^{*}$ as the starting stable configuration, we can then be confident that the energy curvature at $\zeta_{\Gamma(4)}^{*}$ (energy minimum) and at $\zeta_{\Gamma(4)}^{t r}$ (closest energy maximum) will be similar; this implies that both $\zeta_{0}$ and $\zeta_{\Gamma(4)}^{*}$ configurations have similar $\omega_{\Gamma(4)}$ values. Equation 4 is then a plausible approximation of the entire transition path $\zeta_{0} \rightarrow \zeta_{\Gamma(4)}^{t r} \rightarrow \zeta_{\Gamma(4)}^{1}$ connecting the stable state $\zeta_{0}$ with the final state $\zeta_{\Gamma(4)}^{1}$. As an example, we here consider the transition path $\zeta_{0} \rightarrow \zeta_{\Gamma(4)}^{1}$ for the WSe compound along the $\Gamma(4)$ mode. By inspecting Figure 3, we can appreciate

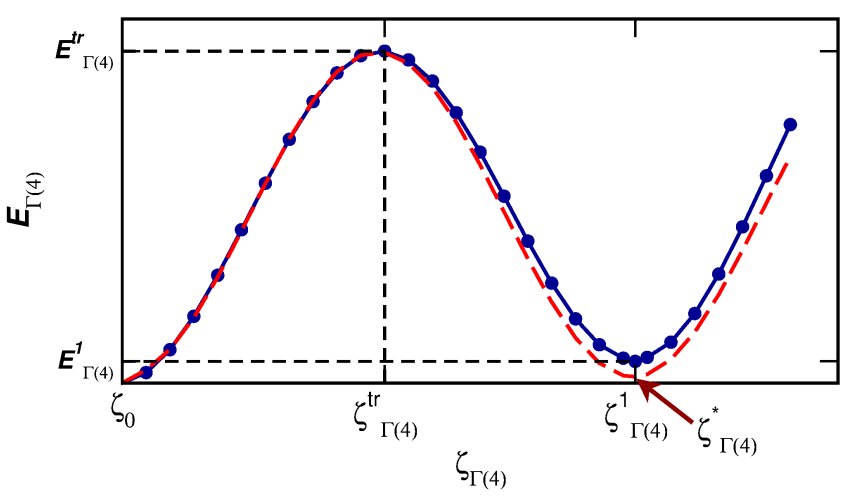

FIG. 3. Blue dots: computed energy values of the WSe system along the $\zeta_{\Gamma(4)}$ configurational coordinate; solid blue line is a guide for the eye. Dashed red line: fit of the computed values by means of Equation 4 in the range $\left[\zeta_{0}, \zeta_{\Gamma(4)}^{t r}\right]$. The position of the energy minimum $\zeta_{\Gamma(4)}^{1}>\zeta_{\Gamma(4)}^{t r}$, obtained from the calculated data, is very close to $\zeta_{\Gamma(4)}^{*}$, the latter obtained as an approximation of $\zeta_{\Gamma(4)}^{1}$ by means of Equation 4.

that the position $\zeta_{\Gamma(4)}^{1}$ of the computed energy minimum $E_{\Gamma(4)}^{1}$ and the position $\zeta_{\Gamma(4)}^{*}$ of the minimum extrapolated from Equation 4 are very close.

According to the presented results, we have been able to individuate a possible transition (layer shift), that the examined TMDs can undergo, by exploiting the knowledge of only one phonon mode.

\section{B. Layer Sliding in $\mathrm{MX}_{2}$ Transition Metal Dichalcogenides}

We now consider again the $\mathrm{MX}_{2}$ and Ti:MoS bulk structures discussed in section III A, and apply the NMTA method to study the energetics of one of the possible layer sliding paths. To build a possible guess for the path, we select the low frequency $\Gamma(4-6)$ and $A(1-6)$ modes, representing layer sliding $(\Gamma(4-5), \mathrm{A}(1-4))$ and inter-layer distance reduction/elongation along a direction orthogonal to the layer plane $(\Gamma(6)$ and $\mathrm{A}(5-6)$, also known as flexural modes); we already pointed at such modes to be related to the layer sliding by means of geometric considerations. ${ }^{8-10}$ For each of the six considered chemistries, we build the guess for a possible $\zeta_{0} \rightarrow \zeta_{1}$ sliding path by starting from the optimized geometry $\left(\zeta_{0} \equiv \zeta=0\right)$ and overlapping the distortion pattern of the selected $\Gamma(4-6)$ and A(1-6) modes at different amplitudes (Figure 4). We then fully relax the $\left[\zeta_{0}, \zeta_{1}\right]$ path using the NEB method, without imposing any constraint on the atomic positions.

To quantify the evolution of the structural distortions along the sliding path, we perform a group-theoretical analysis of each geometry by decomposing the NEBoptimized structures into irreducible representations of the reference geometry at $\zeta=0$, aided by the ISODISTORT ${ }^{15}$ software. Irrespective of the chemical composition, we observe that the unit cell volume is not constant along 
(a)
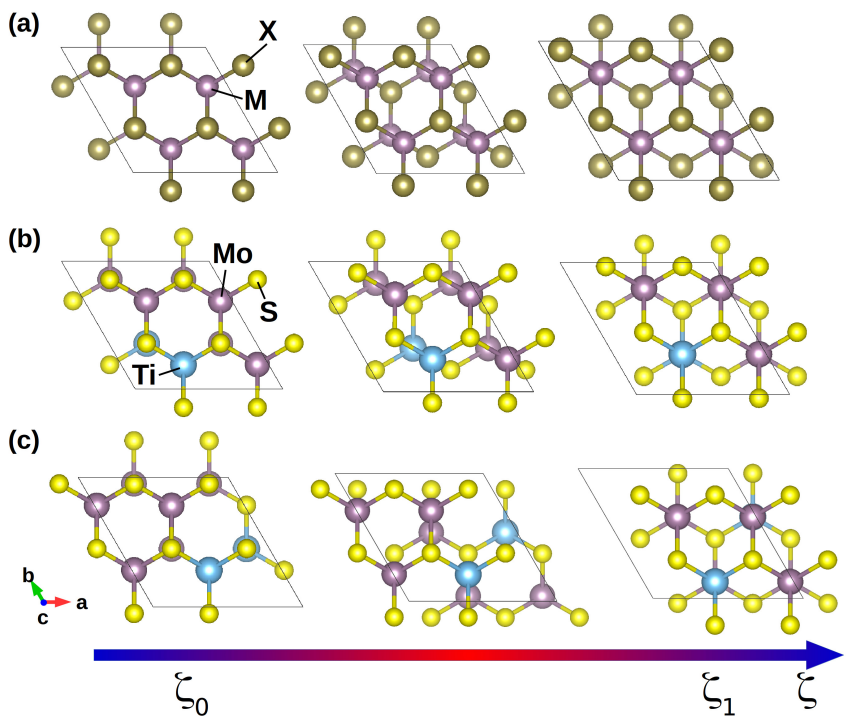

FIG. 4. Schematic representation of the $\zeta_{0} \rightarrow \zeta_{1}$ sliding path, showing only one intermediate configuration as an example. For MX systems (a), only one sliding path has been considered, while for the Ti:MoS system (b-c), two distinct sliding paths have been built. In (b), at $\zeta=\zeta_{1}$, Ti atoms belonging to different layers are aligned along the $\hat{c}$ direction. In (c), at $\zeta=\zeta_{1}$, Ti atoms belonging to one layer are aligned with Mo atoms belonging to adjacent layers along the $\hat{c}$ direction.

the sliding path; moreover, interestingly, we find that the atomic displacement patterns leading the layer sliding can be ascribed to the $\Gamma(4-5)$ and $\Gamma(6)$ modes, representing rigid layer shifts, while intralayer motions have negligible contributions. Following these results, we approximate the calculated energy profiles by means of Equation 6, in which we set $k=\Gamma(4-5), \Gamma(6)$ (Figure 5). We first observe that the Ti:MoS system shows the lowest potential energy barrier along the individuated sliding path; moreover, low $\zeta^{t r}$ structural distortion amplitudes realize low $E^{t r}$ energy maximum values (Figure 6a). Irrespective of the atomic type, the $\Gamma(4-5)$ degenerate modes prevail on the $\Gamma(6)$ one, being the $C_{\Gamma(4-5)}$ amplitudes the main contributions to $\zeta^{t r}$. The energy barrier can thus be lowered by selecting the atomic types that minimize the $C_{\Gamma(4-5)}$ amplitudes needed to realize the energy maximum. To this aim, we relate the $\zeta^{t r}$ amplitude to the M-X pair cophonicity (Table I) corresponding to the $\Gamma(4-5)$ modes (Figure $6 \mathrm{~b})$. We observe that the lowest $\zeta^{t r}$ value is realized when $C_{p h}(\mathrm{M}-\mathrm{X}) \approx 0.0 \mathrm{~cm}^{-1}$; this corresponds to the Ti:MoS system, which is the one showing the lowest energy barrier to overcome in order to realize the individuated $\zeta_{0} \rightarrow \zeta_{1}$ sliding displacement. In this respect, the M-X pair cophonicity can be exploited to select the proper atomic type in order to adjust the energy barrier of specific sliding paths.

These results show that, along the selected sliding path, the sliding barrier is mainly due to the displacement pattern of the low frequency $\Gamma(4-5)$ degenerate modes,

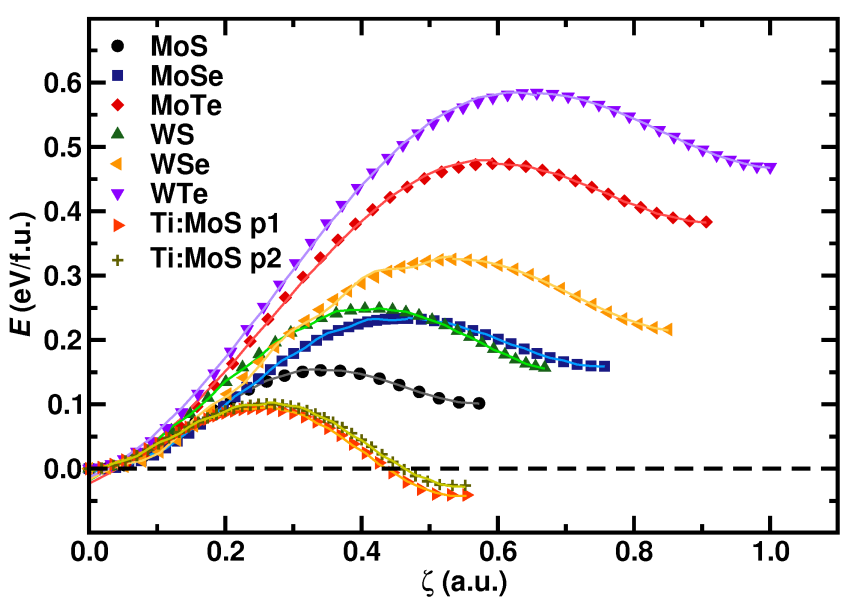

FIG. 5. Energy profile along the configurational coordinate representing the overlap of the $\Gamma(4-5)$ and $\Gamma(6)$ modes. The entire path has been split into two parts in order to account for the asymmetric profile of the energy barrier about the transition state. Filled symbols represent the calculated data while the solid line is the best fit by means of Equation 6 with $\Gamma(4-6), \Gamma(6)$. Data labeled as "Ti:MoS p1" and "Ti:MoS p2" correspond to the paths depicted in Figure $4 \mathrm{~b}$ and Figure $4 \mathrm{c}$, respectively.

corresponding to parallel rigid shifts of subsequent $\mathrm{MX}_{2}$ layers, while other atomic displacements play a minor role. More sliding paths, other than the $\zeta_{0} \rightarrow \zeta_{1}$ just discussed, can be individuated by combining other distortion modes at different amplitudes; each guess can then be optimized and decomposed into mode contributions following the NMTA prescription as discussed above, and the resulting barrier finely adjusted by a proper selection of the atomic type. Indeed, this is one of the main future directions of the present work, expanding, in a systematic way, our investigation on the atomic contributions to the nanoscale friction. The present outcomes finally provide further evidences that our previously reported Ti-doped $\mathrm{MoS}_{2}$ phase is a promising candidate as new material with enhanced tribologic properties.

\section{CONCLUSIONS}

We studied the atomic contributions to the nanoscale friction in layered transition metal dichalcogenides by combining group-theory analysis with ab initio techniques. To this aim, we formulated a method, named NormalModes Transition Approximation, which allowed us to individuate a possible sliding path starting from a stable geometric configuration, by means of the computation of only the phonon spectrum of the stable system. Each displacement pattern relative to specific phonon modes is regarded as a configurational coordinate; possible transition paths can be built by considering linear combinations of such configurational coordinates.

We find that the system energy along such coordinates 

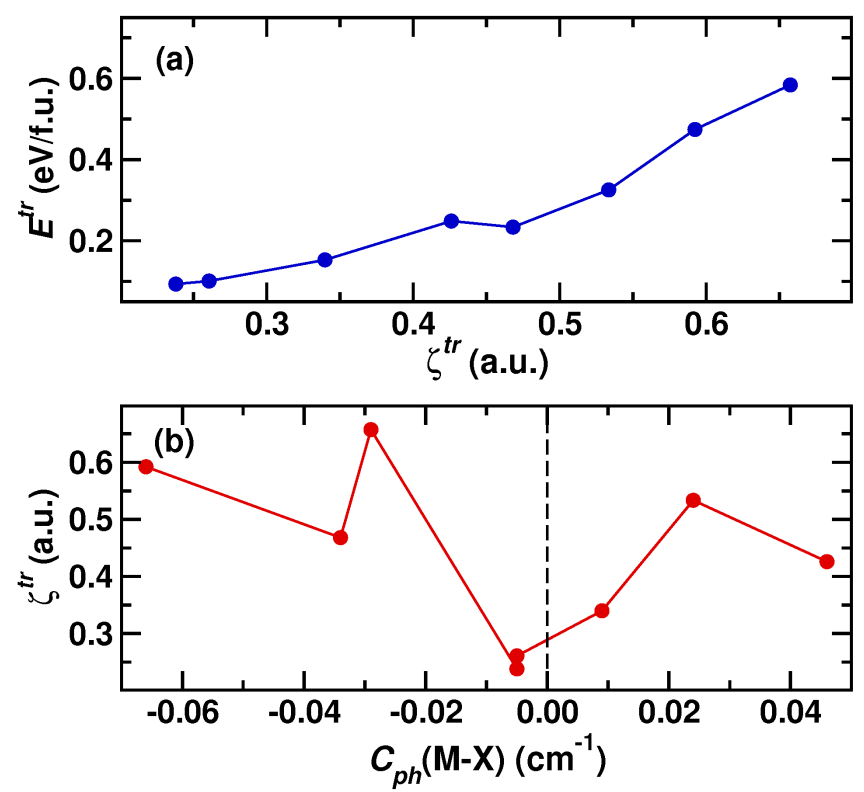

FIG. 6. (a) Energy maximum values, extracted from data shown in Figure 5, as a function of the corresponding $\zeta^{t r}$ distortion amplitude: low sliding barriers are realized at small structural distortions, the latter obtained (b) by selecting the atomic type in such a way that the M-X cophonicity is close to zero. Lines are guides for the eye.

can be approximated by sinusoidal functions of the mode frequency and amplitude; accordingly, energy barriers are functions of the mode frequency and the amount of distortion amplitude needed to build the transition state. By changing the frequency, it is possible to tune the energy barrier and the transition state geometry. In turn, the mode frequency can be tuned by means of electrostructural descriptors.

We apply the Normal-Modes Transition Approximation method to the study of the layer sliding of selected TMD systems. We find that, irrespective of the chemical composition, the atomic displacement pattern leading the sliding motion can be ascribed to the $\Gamma(4-6)$ modes, corresponding to rigid shifts of subsequent layers, while other distortions play a negligible role. Accordingly, lower sliding energy barriers are realized when the cophonicity calculated about the $\Gamma(4-5)$ modes is close to zero; such condition also realizes the minimum sliding mode amplitude among the considered systems. These outcomes furtherly support our suggestion on a previously reported Ti-doped $\mathrm{MoS}_{2}$ phase, as promising novel material with improved frictional properties. Following the NMTA pre-

* cammaant@fel.cvut.cz

$\dagger$ nCATS, FEE, University of Southampton, SO17 1BJ Southampton, United Kingdom

1 K. S. Novoselov, A. K. Geim, S. V. Morozov, D. Jiang, scription, we will extend our exploration on the nanoscale friction to different $\mathrm{MX}_{2}$-derived systems as future works.

We also considered two case studies, namely the inversion of the ammonia molecule and the isomerization of the hydrogen cyanide as case studies, in order to show how the NMTA method is able to decompose energy barriers and reaction rates in terms of normal modes that cooperate to produce the transition path. We find that the energy barrier of the ammonia inversion can be described by the coupling of the stretching and bending mode, the latter leading the geometric advancement of the transition and giving the highest contribution to the transition rate. Concerning the $\mathrm{HCN} \rightarrow \mathrm{CNH}$ isomerization, we observe that the reaction is initiated by the cooperation of the bending and asymmetric bond stretching modes, while the reverse reaction is lead by the coupling of the two stretching modes of the CNH stable isomer. These last results show that the NMTA method also provides information on which phonon modes must be mainly excited to initiate and lead a specific transition.

The Normal-Modes Transition Approximation method is then able to individuate possible transition paths to be used as guess to calculate the minimum energy path by using rare-event sampling methods. It can serve as a support to experimental methods to determine transition states from characteristic patterns in frequencydomain spectra. ${ }^{20-23}$ The method can also be used to tune the energetic and the structural features of specific phonon modes in materials where the electron-phonon coupling plays a fundamental role in the system response to external stimuli, like in electronic or optical device applications, ${ }^{24-28}$ or in energy production/storage materials. ${ }^{29-32}$ Finally, the method can be exploited to guide the deconvolution of vibrational spectra and to study the phonon-phonon coupling in a broad variety of applications. ${ }^{33-37}$

\section{ACKNOWLEDGMENTS}

This work has been done with the support of the Czech Science Foundation, project 17-24164Y. This work was supported by the IT4Innovations Centre of Excellence project (CZ.1.05/1.1.00/02.0070), funded by the European Regional Development Fund and the national budget of the Czech Republic via the Research and Development for Innovations Operational Programme, as well as Czech Ministry of Education, Youth and Sports via the project Large Research, Development and Innovations Infrastructures (LM2011033). The use of VESTA ${ }^{38}$ software is also acknowledged.
Y. Zhang, S. V. Dubonos, I. V. Grigorieva, and A. A. Firsov, Science 306, 666 (2004).

2 A. K. Geim and I. V. Grigorieva, Nature 499, 419 (2013), perspectives. 
${ }^{3}$ RadisavljevicB., RadenovicA., BrivioJ., GiacomettiV., and KisA., Nat Nano 6, 147 (2011).

${ }^{4}$ M. Chhowalla, H. S. Shin, G. Eda, L.-J. Li, K. P. Loh, and H. Zhang, Nat. Chem. 5, 263 (2013).

5 A. Vanossi, N. Manini, M. Urbakh, S. Zapperi, and E. Tosatti, Rev. Mod. Phys. 85, 529 (2013).

6 A. Blumberg, U. Keshet, I. Zaltsman, and O. Hod, The Journal of Physical Chemistry Letters 3, 1936 (2012).

7 G. Levita, A. Cavaleiro, E. Molinari, T. Polcar, and M. C. Righi, J. Phys. Chem. C 118, 13809 (2014).

8 A. Cammarata and T. Polcar, Inorg. Chem. 54, 5739 (2015).

9 A. Cammarata and T. Polcar, RSC Adv. 5, 106809 (2015).

10 A. Cammarata and T. Polcar, Phys. Chem. Chem. Phys. 18, 4807 (2016).

11 C. Dellago, P. G. Bolhuis, and D. Chandler, J. Chem. Phys. 110, 6617 (1999).

12 G. Henkelman and H. Jónsson, J. Chem. Phys. 113, 9978 (2000).

13 J. S. H. Goldstein, C. Poole, Classical Mechanics, 3rd ed. (Pearson Education, 2002) p. 238, chapter 6, "Oscillations".

14 See Supplemental Material at http://xxx.xxx, section "The $E_{k}$ Sinusoidal Form Hypothesis".

15 B. J. Campbell, H. T. Stokes, D. E. Tanner, and D. M. Hatch, J. Appl. Cryst. 39, 607 (2006).

16 D. Orobengoa, C. Capillas, M. I. Aroyo, and J. M. PerezMato, J. Appl. Crystallogr. 42, 820 (2009).

17 J. M. Perez-Mato, D. Orobengoa, and M. I. Aroyo, Acta Crystallogr. A 66, 558 (2010).

18 Example geometries at particular amplitude values are reported in the " $\Gamma(4)$ Rigid-layer Shift Vibrational Mode of MX and Ti:MoS Systems" sections of the Supplemental Material at http://xxx.xxx.

19 W. Setyawan and S. Curtarolo, Comp. Mater. Sci 49, 299 (2010).

20 T. P. Dougherty, G. P. Wiederrecht, K. A. Nelson, M. H. Garrett, H. P. Jensen, and C. Warde, Science 258, 770 (1992).
21 H. S. Chung, K. McHale, J. M. Louis, and W. A. Eaton, Science 335, 981 (2012).

${ }^{22}$ H. S. Chung, S. Piana-Agostinetti, D. E. Shaw, and W. A. Eaton, Science 349, 1504 (2015).

23 J. H. Baraban, P. B. Changala, G. C. Mellau, J. F. Stanton, A. J. Merer, and R. W. Field, Science 350, 1338 (2015).

${ }^{24}$ M. Imada, A. Fujimori, and Y. Tokura, Rev. Mod. Phys. 70, 1039 (1998).

25 X.-L. Zhang and W.-M. Liu, Sci. Rep. 5, 8964 (2015).

26 S. Johnston, C. Monney, V. Bisogni, K.-J. Zhou, R. Kraus, G. Behr, V. N. Strocov, J. Malek, S.-L. Drechsler, J. Geck, T. Schmitt, and J. van den Brink, Nat. Commun. 7, 10563 (2016).

27 J.-H. Yang, L. Shi, L.-W. Wang, and S.-H. Wei, Sci. Rep. 6, 21712 (2016).

${ }^{28}$ H. Boschker, C. Richter, E. Fillis-Tsirakis, C. W. Schneider, and J. Mannhart, Sci. Rep. 5, 12309 (2015).

${ }^{29}$ L. Waldecker, R. Bertoni, R. Ernstorfer, and J. Vorberger, Phys. Rev. X 6, 021003 (2016).

30 A. Cammarata, P. Ordejón, A. Emanuele, and D. Duca, Chem-Asian J. 7, 1827 (2012).

31 A. Cammarata, A. Emanuele, and D. Duca, Chem-Asian J. 7, 1838 (2012).

32 A. Cammarata, A. Martorana, and D. Duca, J. Phys. Chem. A 113, 6381 (2009).

33 L. Fan, K. Y. Fong, M. Poot, and H. X. Tang, Nat. Commun. 6, 5850 (2015).

34 V. J. Gokhale and M. Rais-Zadeh, Sci. Rep. 4, 5617 (2014).

35 R. Su, Z. Yuan, J. Wang, and Z. Zheng, Sci. Rep. 6, 19628 (2016).

36 L. Lindsay, D. A. Broido, and N. Mingo, Phys. Rev. B 80, 125407 (2009).

37 C. W. Li, J. Hong, A. F. May, D. Bansal, S. Chi, T. Hong, G. Ehlers, and O. Delaire, Nat. Phys. 11, 1063 (2015).

38 K. Momma and F. Izumi, J. Appl. Cryst. 41, 653 (2008). 